\title{
Exhaust Manifold Design - FEA Approach
}

\author{
Gopaal $^{\# 1}$, MMM Kumara Varma ${ }^{* 2}$, Dr L Suresh Kumar ${ }^{\# 3}$ \\ ${ }^{\# 1}$ Assistant Professor, Ramanandatirtha Engineering College, Nalgonda, Telangana, India \\ 2 Assistant Professor, Sri Sivani College of Engineering, Chilkapalem, Srikakulam, Andhra Pradesh, India \\ 3 Principal, Ramanandatirtha Engineering College, Nalgonda, Telangana, India
}

\begin{abstract}
The Exhaust manifold in the engines is an important component which has a considerable effect on the performance of the I.C engine. The exhaust manifold operates under high temperature and pressure conditions. Their design usually has to be performed by trial and error through many experiments and analyses. Therefore, an automated design optimization would reduce technical, schedule, and cost risks for new engine developments.

This paper deals with the various factors that are to be considered in the design of the exhaust manifold. It tries to explain the effect of various factors during the Finite Element Analysis.
\end{abstract}

Keywords - Exhaust manifold, Thermal analysis, Structural analysis, Couple Field analysis, Wave rarefaction, Mass Participation factor.

\section{INTRODUCTION}

The exhaust manifold used in a 4-stroke IC engine is mounted on the cylinder head of an engine. The gases exhausted from the engine are collected by the exhaust manifold and it is sent to a catalyst converter. The exhaust manifold has an important role in the performance of an engine system. The exhaust manifold affects the efficiencies of emission and fuel consumption.

During the process of collecting the gas from engine and exhaust to catalyst converter, the exhaust manifold experiences temperatures of $800^{\circ} \mathrm{c}$ and the pressures varying from 100 to 500 kpa.

The exhaust manifold is under a thermal fatigue produced by the exhaust gases increasing and decreasing temperatures. The pressure waves of the emitted exhaust gases during particular times of the cycle subject internal pressure. These will lead to cracks in the exhaust manifold.

Thermal and mechanical loadings are the major factors in the failure of the exhaust manifold. To maximize the engine power at high speeds, the exhaust gases should be vented out smoothly from the piston chambers to the exhaust manifold.

Structural and thermal analysis is performed with an internal pressure load, and stresses and deflections are documented. Efforts are made to optimize the design for the above said conditions.

A great deal of efforts are done to increase the performance and to reduce the weight. Automotive companies are trying to achieve a goal in optimal engine design.

\section{LITERATURE REVIEW}

Bin Zou, et al. have analysed the impact of temperature effect on exhaust manifold modal analysis. From the CFD analysis temperature field is mapped and then heat conduction process is analysed in FEM software with the temperature field boundary conditions. Lastly the modal analysis that considers temperature effect is done. On comparison of the frequency and vibration mode between cold modal and thermal modals it is observed that the temperature has a great influence on the manifold mode, which is very valuable for the product design.

Sweta Jain, et al. in their paper "The Sequential Coupled Thermal Structural Analysis" have investigated the associated thermal stresses and deformations under simulated operational conditions close to the real situation on different materials at different ambient temperatures i.e. cast iron, structural steel. The linear steady state temperature distribution under the thermal field \& structural analysis is calculated. The purpose of this analysis is to ensure the appropriateness of material for the defined design from the view point of serviceability of the exhaust manifold.

J.David Rathnaraj in his paper has analysed the thermo mechanical fatigue of stainless steel exhaust manifolds. Automotive engine has large thermal stress induced by temperature gradient and geometrical constraints. This thermo mechanical coupling is one of critical problems in automotive 
engineering. Thermal fatigue analysis is considered in the design process of the exhaust manifold.

The paper focuses on the application of constitutive equation to the thermo mechanical condition of a model based on isothermal data. Using the proposed model, the thermal stress analysis and life prediction of exhaust manifold made of 429EM stainless steel is done.

A.K.M. Mohiuddin, et al. in their paper "Optimal design of automobile exhaust system using gt- power", designed exhaust system using GT-Power software and compared its performance with an existing system. The newly designed exhaust manifold shows lower back pressure which ultimately results better performance of the engine.

Swathi Satish, et al. paper does a comparative study of predictions obtainable using the conformal and indirect interfaces for Conjugate Heat Transfer (CHT) analysis in an exhaust manifold with a view to ascertain the degree of closeness of certain predefined physical quantities. A steady-state analysis was performed for base mesh size values of $2 \mathrm{~mm}$ and $4 \mathrm{~mm}$ using the RANS k-epsilon turbulence model. Predicted results for the conformal and non conformal meshes at the two base mesh size values were observed to be in good agreement.

\section{EXHAUST MANIFOLD DESIGN CONSIDERATIONS AND CRITERIONS}

Present day engines are required to have more engine power and are also required to meet the strict pollution standards. To improve exhaust system performance, many design specifications are required.

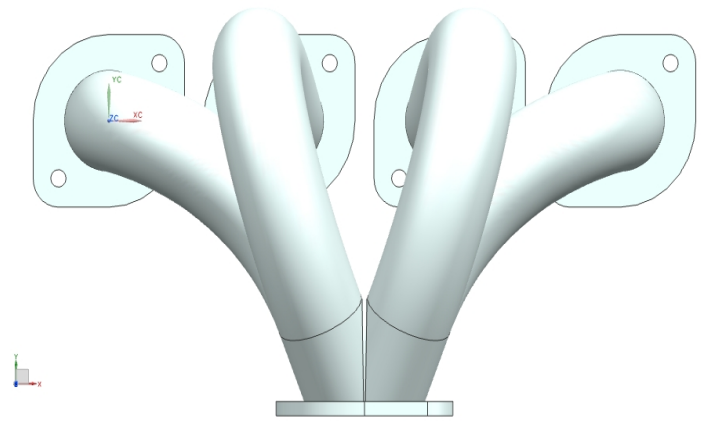

Fig 1. Top view of the exhaust manifold

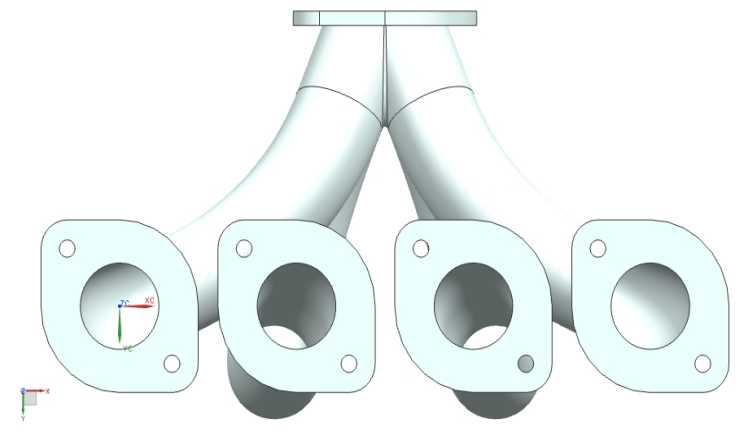

Fig 2. Bottom view of the exhaust manifold

Exhaust gases should be led from the piston chambers to the exhaust manifold smoothly. This will ensure to maximize the engine power at high speeds.

The catalyst will absorb more pollutant at high temperatures. Hence, the exhaust gases should be kept at high temperature in the exhaust pipe even during low speeds.

The exhaust system has to be "tuned" for optimal efficiency. A tuned exhaust system should have geometry which will reflect the pressure waves of the exhaust gases which are emitted, as per engine firing order.

During the exhaust stroke, the piston moves up and the total volume in the cylinder decreases. The exhaust valve opens and lets the high pressure gas from the cylinder to the exhaust manifold. This will create an 'exhaust pulse'. The 'exhaust pulse' will have High, Medium and Low pressure heads.

a) High-pressure head - There is a large pressure difference in the cylinder and outside of the exhaust system.

b) Medium pressure head - The exhaust gas pressure in the cylinder and the outside exhaust system get equalized. The pressure difference and the exhaust velocity decrease.

c) Low pressure head - The exhaust gas pressure initially matches the ambient atmospheric pressure. The momentum of the high- and medium- pressure heads reduce the pressure in the cylinder to a less than atmospheric level. 
The combustion products from the cylinder are extracted due to this low pressure head. It also helps to induct fresh charge during the inlet and outlet overlap period. The effect is known as 'scavenging'. Scavenging is influenced by the length, crosssectional area and shape of the exhaust ports and pipe works.

The scavenging effect is decreased by too large tubes. Big diameter pipes will cause the exhaust gases to expand and slow down. Tubes which are too small will cause resistance to exhaust gas flow. The engine must work more to expel the exhaust gas from the chamber. This will reduce the useful available power and cause dilution of the incoming intake charge. The headers are to be tuned to a particular speed range, as more exhaust gases are produced at higher engine speeds.

It is observed that at higher engine speeds, wide primary tubes offer best gains in power and torque. At lower speeds, narrow tubes are the best.

To improve the scavenging effect, headers are resonance tuned to utilize the low-pressure reflected wave rarefaction pulse. When a change in gas density occurs, wave rarefaction pulses are created. The wave rarefaction pulse is timed to coincide with the valve overlap time. This is done by tuning the length of the primary tubes by resonance tuning. Long primary tubes resonate at lower engine speeds.

It is challenging to design engine meeting the emission norms and the demanded engine drivability. Alternate combustion modes such as homogeneous charge compression ignition (HCCI), low temperature combustion (LTC) and premixed controlled compression ignition (PCCI) are good in reduction of the engine emissions levels. But, complex, precise control systems and technologies are required for making these new modes working. They need different fuelling strategies and incylinder conditions.

New emissions norms are faced effectively by traditional approaches such as Air-to-fuel ratio and dual-loop exhaust gas recirculation (EGR), with both high and low-pressure re circulations.
Usually exhaust gases from the cylinders is collected into one pipe in the manifold assembly. If the gases are not vented out properly it affects the engine performance. A back pressure is created, when the exhaust gases from one cylinder is not completely vacated and exhaust gases released from other cylinders enter the manifold. This happens as the cylinders fire as per the firing order at different times and the exhaust gases leaves the cylinders and enters the exhaust system. Cast iron is preferred material for manifolds as they have the least production cost and has ease of manufacture.

Modern exhaust headers come with a ceramic coating. This coating helps to prohibit rust and also reduces the amount of heat radiated into the surroundings. The engine room is prevented from getting heated and which causes the air entering the engine to have low temperature.

\section{FINITE ELEMENT ANALYSIS CONSIDERATIONS}

\section{THERMAL ANALYSIS:}

Thermal analysis calculates the temperature distribution; amount of heat gained or lost, thermal gradients and thermal fluxes.

\section{TYPES OF THERMAL ANALYSIS}

1. Steady-state thermal analysis is done to determine the temperature distribution and other thermal quantities. The loading conditions are steady-state, wherein the temperature changes over time period is not considered.

2. Transient thermal analysis is done to determine the temperature distribution and other thermal quantities under conditions that vary over a period of time.

\section{STRUCTURAL ANALYSIS}

Structural analysis incorporates the fields of mechanics and dynamics as well as many theories of failure. The loads acting on the structure, geometrical features and support conditions are to be considered for doing structural analysis. The material properties of the exhaust manifold have to be specified. The reactions at the supports, displacement values and stress values are obtained. 
International Journal of Engineering Trends and Technology (IJETT) - Volume17 Number 10-Nov2014

The Von Mises stress values obtained should be less than the yield strength of the material.

\section{COUPLE FIELD ANALYSIS}

Coupled field analysis is done for thermalstructural, wherein the result values obtained of one analysis is used for doing another analysis. The entire model will have single set of nodes. The geometry created for the first analysis is used for the coupled analysis also. The geometry is kept constant throughout.

Create the geometry in the Thermal Environment, where the thermal effects will be applied. The geometry must remain constant, but the element types can change. Thermal elements are required for a thermal analysis while structural elements are required to determine the stress in the structure. A coupled-field analysis can use matrixcoupled ANSYS elements, or sequential loadvector coupling between separate simulations of each phenomenon.

From the Couple field analysis results, the maximum deflection for the loading condition and the Von Mises stresses are obtained.

According to the Maximum Yield Stress Theory, the design under static conditions is considered to be safe when the Von Mises stress are lower than the yield strength of the material.

\section{DYNAMIC ANALYSIS}

Exhaust manifold is analyzed for dynamic loading conditions. To check the structure response for resonance condition, modal analysis is performed on exhaust manifold. The natural frequencies occurring in the operating range is calculated.

\section{MODAL ANALYSIS}

The vibration characteristics such as natural frequencies and mode shapes in the frequency range are found out from Modal analysis. The output serves as a starting point for doing further dynamic analysis such as transient dynamic analysis, harmonic response analysis, etc.

From the modal analysis, a total of 6 natural frequencies are observed in the frequency range. The mass participation of each of these 6 frequencies is listed. The mode shapes of these frequencies are plotted. The maximum mass participation in $\mathrm{X} / \mathrm{Y} / \mathrm{Z}$ direction at different frequencies is also identified.

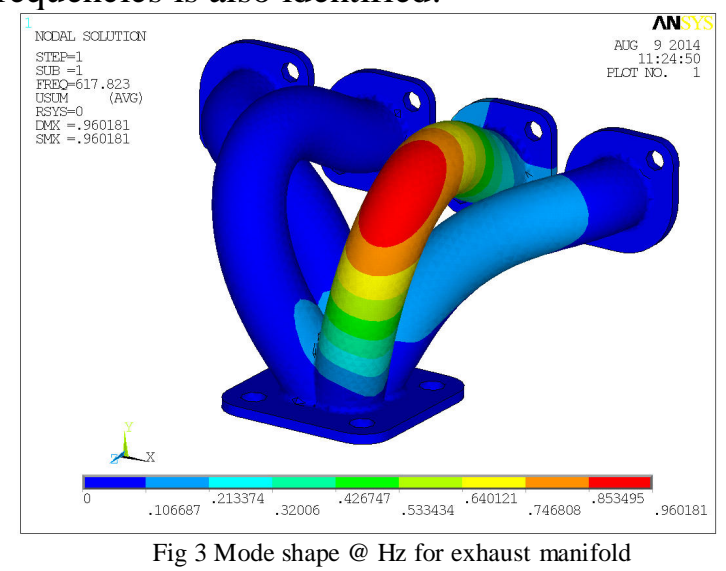

\section{HARMONIC ANALYSIS}

In a structural system, sustained cyclic loads will produce a harmonic response. The dynamic behaviour of the structures is predicted by Harmonic response analysis. This will enable to verify whether the designs will be able to overcome resonance, fatigue, and the other effects of forced vibrations.

In Harmonic response analysis, the steady-state response of a linear structure to loads that vary harmonically with time is determined. The structure's response at several frequencies is observed. A graph of response quantity like displacements versus frequency is drawn. "Peak" responses are identified from the graphs. At these peak frequencies stresses are calculated.

Modal analysis is done to obtain the structure's natural frequency. When the forcing frequency matches with the natural frequency of the structure Harmonic response occurs.

To completely specify a harmonic load, the following information is required: the amplitude, the phase angle, and the forcing frequency range.

From the graphs, the amplitudes are observed. The deflections and stresses nearest to the above frequencies are plotted. They are to be lower than the allowable limits. 


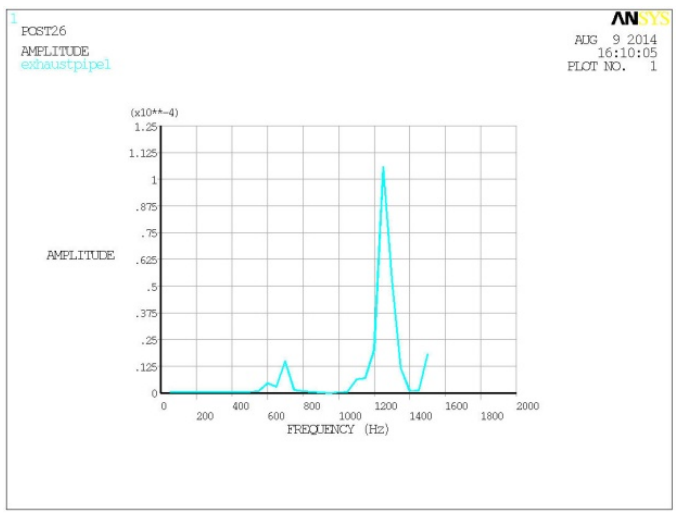

Fig 4 Harmonic location at pipe location

From the above analysis it is to be verified that the exhaust manifold has stresses and deflections within the design limits of the material used. The deflections and stresses obtained in the harmonic analysis are also to be under the design limits of the material.

Then the design can be considered as safe.

\section{FUTURE WORK}

An optimization in the manifold design can be obtained by performing computational fluid dynamics analysis.

\section{ACKNOWLEDGMENT}

The support extended by the Ramanandatirtha Engineering College and Sri Sivani colleges of engineering authorities is highly appreciated and acknowledged with due respect.

\section{REFERENCES}

1. Swathi Satish, Mani Prithiviraj and Sridhar Hari, "Comparison of predictions obtained on an exhaust manifold analysis using conformal and indirect mapped interface", International Congress on Computational Mechanics and Simulation, IIT Hyderabad, 10 12 December 2012.

2. J.David Rathnaraj, "Thermo mechanical fatigue analysis of stainless steel exhaust manifolds", IRACST - Engineering Science and Technolgy, Vol 2, No. 2, April 2012, PP 265 - 267.

3. M.Rajasekhar Reddy, Dr K.Madhava Reddy, "Design And Optimization Of Exhaust Muffler In Automobiles", International
Journal of Engineering Research and Applications, Vol 2, Issue 5, Sept-Oct 2012, PP 395 - 398.

4. Sweta Jain, AlkaBani Agrawal, "Coupled Thermal - Structural Finite Element Analysis for Exhaust Manifold of an Off-road Vehicle Diesel Engine", International Journal of Soft Computing and Engineering, Vol 3, Issue 4, September 2013, PP 226-230.

5. Bin Zou, Yaqian Hu, Zhien Liu,Fuwu Yan and Chao Wang, "The Impact of Temperature Effect on Exhaust Manifold Thermal Modal Analysis", Research Journal of Applied Sciences Engineering and Technology, August 20, 2013, PP 2824-2829.

6. A.K.M. Mohiuddin, Ataur Rahamn and Mohd. Dzaidin, "Optimal design of automobile exhaust system using gt- power", Interantional Journal of Mechanical and Materials Engineering, Vol 2 No. 1, 2007, PP 40 - 47.

7. Modelling and Design of Exhaust Manifold Under Thermo mechanical Loading by K.H. Park, B.L. Choi, K.W. Lee, K.-S. Kimand Y.Y. Earmme.

8. Masahiro Kanazaki, Masashi Morikawa, "Multiobjective Design Optimization of Merging Configuration for an Exhaust Manifold of a Car Engine", Parallel Computational Fluid Dynamics, International Conference Parallel, Kyoto, Japan, May 2002.

9. Exhaust Manifold Pressure Estimation Diesel Equipped with a VGT Turbocharger by Felipe Castillo and Emmanuel Witrant, Luc Dugard.

10. Practical Finite Element Analysis by Nitin S Gokhale, Sanjay S Deshpande, Sanjeev V Bedekar and Anand N Thite. 